\title{
Heartbeat: can machine learning improve outcomes in patients with heart failure with preserved ejection fraction?
}

Machine learning offers the opportunity to gain new insights into clinical cardiovascular disease as shown in the paper in this issue of Heart on novel patient phenogroups in heart failure with preserved ejection fraction (HFpEF). ${ }^{1}$ Model-based clustering was performed based on echocardiographic, clinical and laboratory variables (including proteomics) in 320 outpatients (mean age 78 years, $56 \%$ female) with HFpEF to identify six phenogroups with significant differences between groups in the composite outcome of all-cause mortality or heart failure hospitalisation at up to $2 \frac{1}{2}$ years follow-up. The highest event rates occurred in phenogroup 2, characterised by older age, reduced right ventricular function, atrial fibrillation in $85 \%$, hypertension in $83 \%$ and chronic obstructive pulmonary disease in 30\%. Poor outcomes were also seen in phenogroup 1 which was defined by the presence of hypertension, coronary disease, renal disease, diabetes and anaemia (figure 1).

In the accompanying editorial, Chen and Banerjee $^{2}$ provide a brief summary of the approach to using machine learning (ML) in research with the goal of improving patient care. A Cardiology in Focus article ${ }^{3}$ explains the relationship between artificial intelligence, machine learning and deep learning as these terms are now defined (figure 2). In their editorial, Chen and Banerjee argue that: 'Consensus guidelines for ML in research and clinical practice are urgently required if these tools are going to translate to patient care. External validation in research studies using ML in healthcare will help to understand which clustering and prediction tools are of greatest use to the data, and suitable for clinical implementation. In order for ML to create patient benefit, the investigations need to shift from the frameworks of discovery science to evidence-based healthcare and implementation science.'

Efforts to shorten the time between occurrence of an ST-elevation myocardial infarction (STEMI) and effective treatment primarily have focused on

Correspondence to Professor Catherine M Otto, Division of Cardiology, University of Washington, Seattle,WA 98331, USA; cmotto@uw.edu

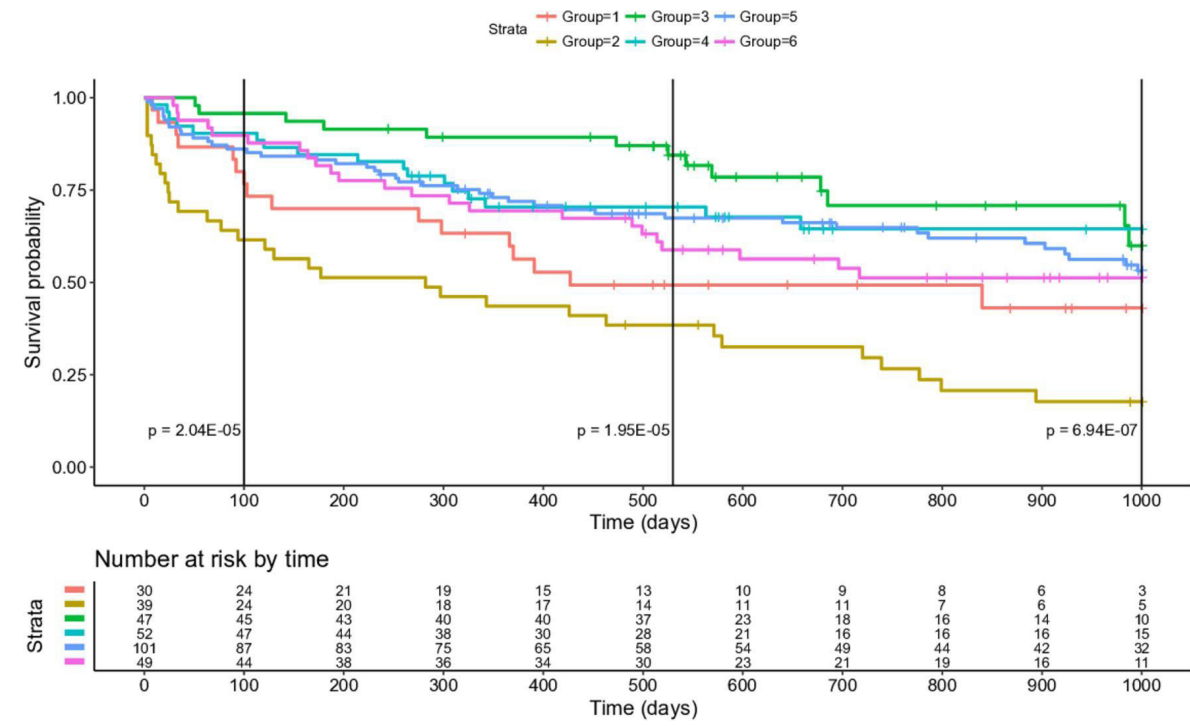

Figure 1 Kaplan-Meier curves of composite end points during 1000 days of follow-up from stable condition for phenogroups. The log rank $p$ values for the composite end point at an early time point (100 days) and a mid-range time point (18 months) are also shown. door-to-balloon time. The importance of the time from first medical contact (FMC) to door time has received less attention. In a meta-analysis of over 100 studies conducted in 20 countries that included over 125000 patients, Alrawashdeh and colleagues ${ }^{4}$ found that each $10 \mathrm{~min}$ increase in the FMC-to-door time was associated with a $10.6 \%(95 \%$ CI $7.6 \%$ to $13.5 \% ; \mathrm{p}<0.001)$ reduction in the proportion of patients treated within $90 \mathrm{~min}$. The marked variation between countries in FMC-to-door time (figure 3) which is not simply explained by geography or transport options, suggests there is room for improvement

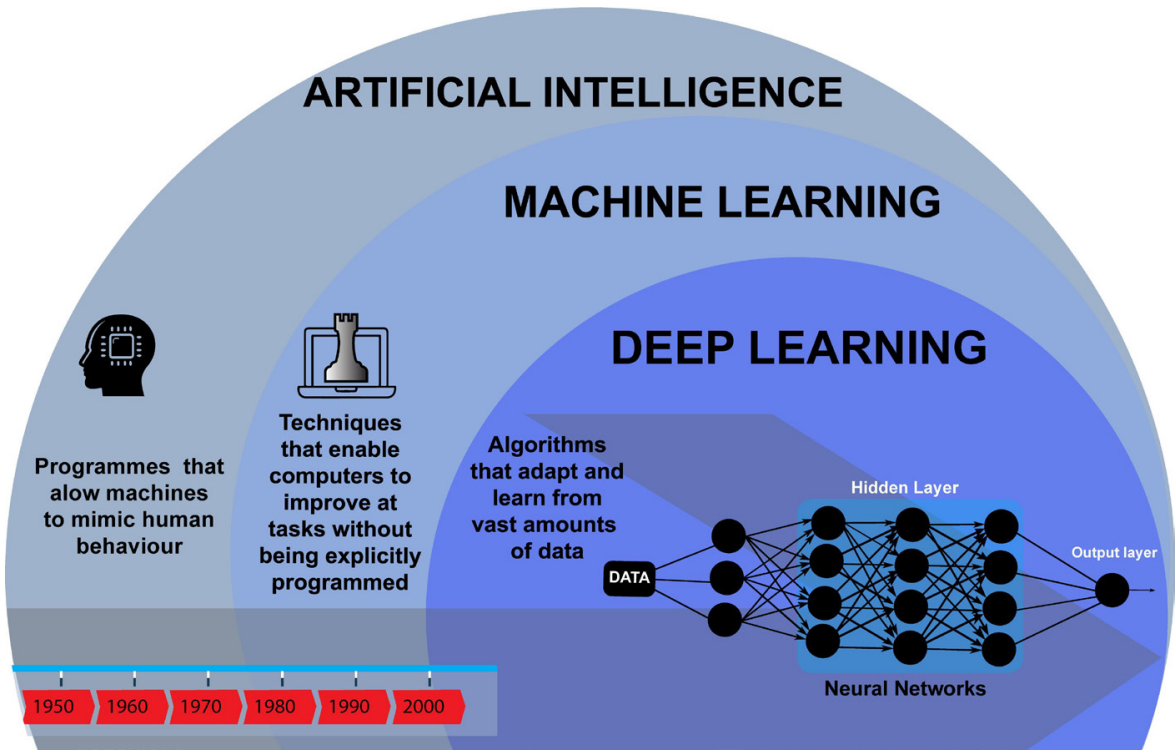

Figure 2 Artificial intelligence through time. 


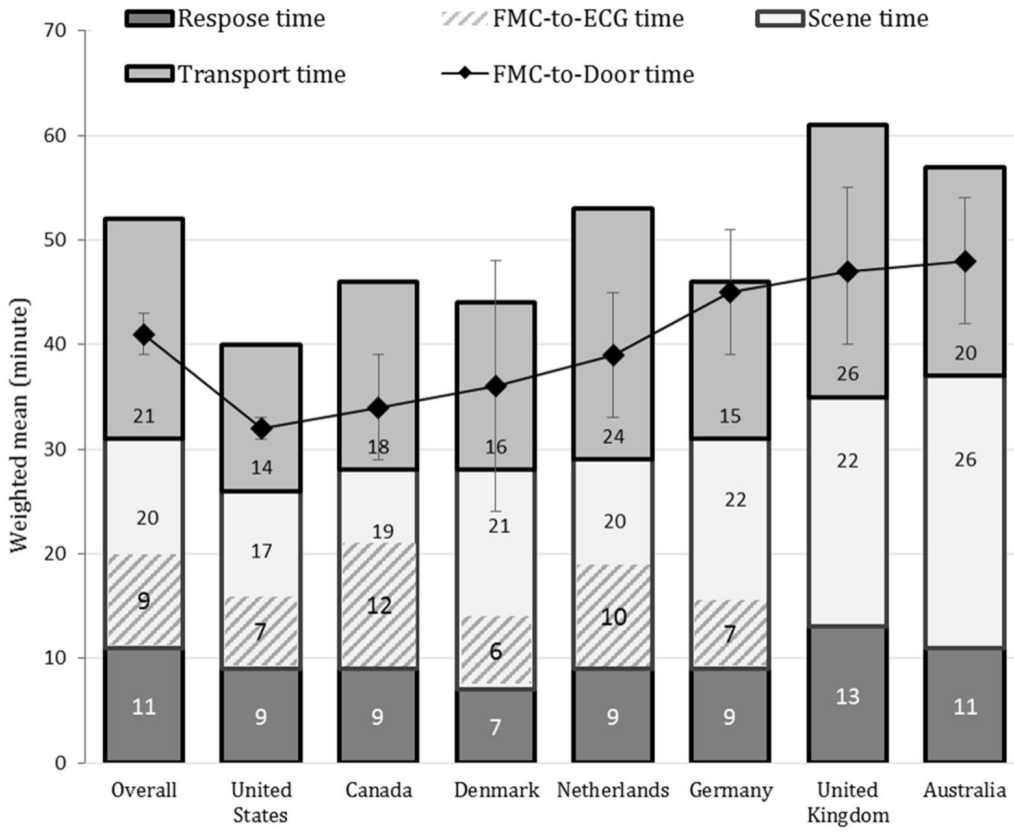

Figure 3 FMC-to-door time and other EMS components among countries. EMS, emergency medical services; FMC, first medical contact.

in the worldwide care of patients with STEMI.

Marcolino and Ribeiro ${ }^{5}$ point out that 'since these studies are predominantly from urban areas in high-income countries, an additional challenge would be to understand this relation in rural and remote areas, and in low- and middleincome countries (LMICs), as Brazil or China, since most of STEMI deaths occur in LMICs. Due to the limitations in access to specialised care and organisational factors such as lack of a formal EMS-based network, dirt roads and paths with ferry crossings, we could infer that this relation between distance and time is probably much higher than the observed in these other scenarios.'

Another research study in this issue of Heart addresses the importance of
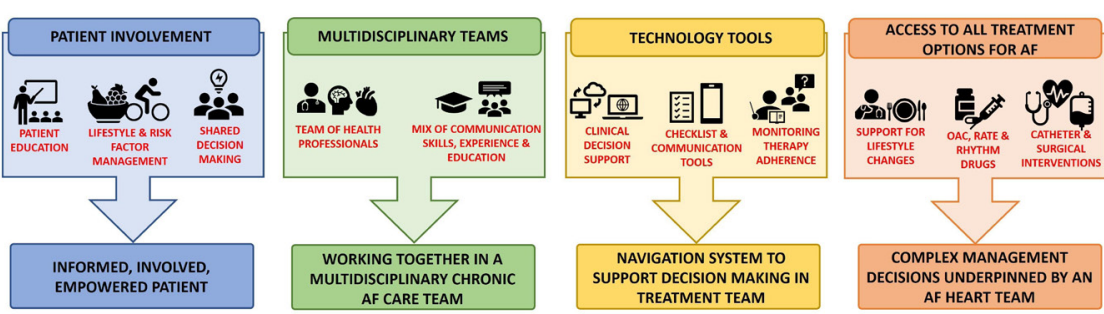

FUNDAMENTALS OF TEAM-BASED INTEGRATED CARE MODEL IN AF

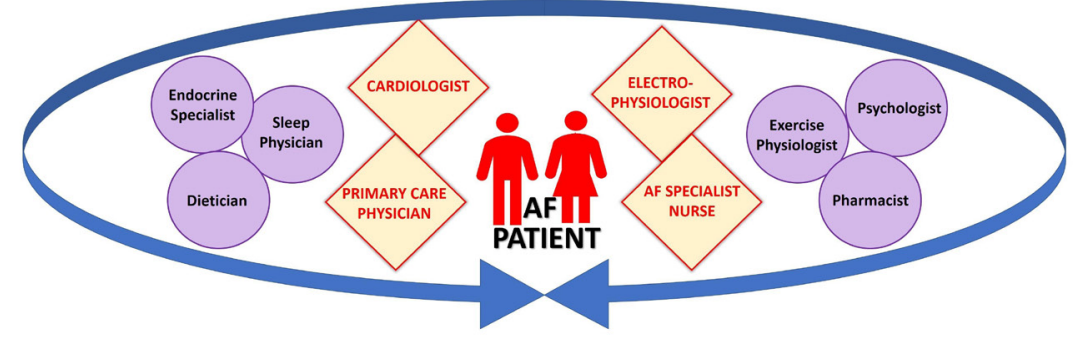

Figure 4 Integrated care approach: the fundamentals of integrated care involve four key elements. Patient involvement, multidisciplinary teams, technology tools and access to all treatment options for atrial fibrillation (AF). These are delivered by a team of healthcare practitioners who work closely with the patient to improve AF outcomes. adjusting non-vitamin-K antagonist oral anticoagulation (NOAC) dosage in patients with a decline in renal function. ${ }^{6}$ In this cohort of 4120 patients with atrial fibrillation treated with a NOAC, although a significant decline in renal function occurred in about $4 \%$ over 2 years of follow-up, few (about 20\%) had their NOAC dose adjusted, which was associated with an increased risk of major bleeding and bleeding hospitalisation. Yao and Noseworthy ${ }^{7}$ discuss the complexities of NOAC dosing, patient compliance, monitoring and potential drug interactions. They propose that the infrastructure and expertise of 'coumadin clinics' be repurposed to encompass all types of oral anticoagulation.

The review article on lifestyle modifications for treatment of atrial fibrillation $^{8}$ in this issue of Heart will be of great value to clinicians. Rather than just focusing on rhythm, rate control and anticoagulation, we should work more closely with our patients on modifiable risk factors including hypertension, sleep apnea, diabetes and hyperlipidaemia as well as health behaviours such as maintaining a normal body weight, exercising, not smoking and avoiding excessive alcohol consumption. The authors propose an integrated care process focused on the patient using technology tools within a multidisciplinary team approach figure 4 .

\section{Contributors All authors contributed.}

Funding The authors have not declared a specific grant for this research from any funding agency in the public, commercial or not-for-profit sectors.

Competing interests None declared.

Patient consent for publication Not required.

Provenance and peer review Commissioned; internally peer reviewed.

(C) Author(s) (or their employer(s)) 2020. No commercial re-use. See rights and permissions. Published by BMJ.

\section{(D) Check for updates}

To cite Otto CM. Heart 2020;106:315-317.

Heart 2020;106:315-317.

doi:10.1136/heartjnl-2020-316635

\section{ORCID iD}

Catherine M Otto http://orcid.org/0000-0002-05279392

\section{REFERENCES}

1 Hedman Åsa K, Hage C, Sharma A, et al. Identification of novel pheno-groups in heart failure with preserved ejection fraction using machine learning. Heart 2020;106:342-9.

2 Chen S, Banerjee A. Machine learning: a long way from implementation in cardiovascular disease. Heart 2020;106:318-20. 
3 de Marvao A, Dawes TJ, Howard JP, et al. Artificial intelligence and the cardiologist: what you need to know for 2020. Heart 2020;106:399-400.

4 Alrawashdeh A, Nehme Z, Williams B, et al. Emergency medical service delays in ST-elevation myocardial infarction: a meta-analysis. Heart 2020;106:365-73.
5 Marcolino MS, Ribeiro T. Focusing on prehospital care to improve ST elevation myocardial infarction care. Heart 2020;106:323-4.

6 Inohara T, Holmes DN, Pieper K, et al. Decline in renal function and oral anticoagulation dose reduction among patients with atrial fibrillation. Heart 2020;106:358-64.
7 Yao $X$, Noseworthy P. NOAC dosing and monitoring: really as simple as it seems? Heart 2020;106:321-2.

8 Sanders P. Lifestyle modifications for treatment of atrial fibrillation. Heart 2020;106:325-32. 